Tropical Journal of Pharmaceutical Research April 2010; 9 (2): 110-118

(c) Pharmacotherapy Group, Faculty of Pharmacy, University of Benin, Benin City, 300001 Nigeria.

All rights reserved.

Research Article

Available online at http://www.tjpr.org

\title{
Documentation on Medicinal Plants Sold in Markets in Abeokuta, Nigeria
}

\author{
MacDonald Idu*, Joseph O Erhabor and Harriet M Efijuemue \\ Department of Plant Biology and Biotechnology, University of Benin, PMB II54, Benin City, Nigeria
}

\begin{abstract}
Purpose: To document the medicinal values, local names, method of preparation, dosage forms and parts of common medicinal plants sold in some markets in Abeokuta, Nigeria.

Methods: Ethnobotanical data were collected by oral interview and with the aid of a structured questionnaire administered to men, women and young girls. The data from respondents from the five local markets surveyed were documented.

Results: The results show that a total of 60 medicinal plant species are commonly used by the people of Abeokuta in their traditional health care system. Most of the plant materials were in dried form and sold singly or in combination with other plants and are used in the treatment of various ailments such as malaria, hypertension, typhoid, jaundice, hyperthermia, skin irritations, dysentery, anaemia, gonorrhea, cough, measles and fibroid. A majority of the plants were trees. The respondents were women (64.3\%), young girls $(28.5 \%)$ and men (7.14\%).

Conclusion: The findings support the need to encourage domestication and cultivation of medicinal plants as well as put in place conservation measures to ensure sustainable source of medicinal plants.
\end{abstract}

Keywords: Ethnomedicine; Medicinal plants cultivation; Documentation; Traditional medicine. 


\section{INTRODUCTION}

Plants have been major sources of medicine and plant secondary metabolites has been attributed for most plants' therapeutic activities [1,2]. Phytomedicines have shown great promise in the treatment of intractable infectious diseases [3]. The local uses of plants and products in health care are even much higher particularly in those areas with little or no access to modern health services [4].

Traditional medicine practice is an important part of healthcare delivery system in most of the developing world [5] and is a source of primary health care to $80 \%$ of the world's population [6]. Traditional medicine has been reported to be the first medical care known to Nigerians and herbal medicine, an aspect of traditional medicine, is becoming increasingly popular in both developing and developed countries [7]. Traditional medical knowledge of medicinal plants and their use by indigenous culture are not only useful for conservation of cultural traditions and biodiversity but also for community healthcare and drug development now and in the future $[8,9]$

Global and national markets for medicinal herbs have been growing rapidly and significant economic gains are being realized with global sales of herbal products which totalled an estimated US $\$ 60$ million in 2000 [10]. Local markets form an integral part of the life and culture of the people of Abeokuta, Nigeria. The markets are also important socio-economic institutions. The traders in these markets sell large amounts of medicinal plants to the indigenes and foreigners who seek their help. Most of the plant parts (barks, roots, stems and leaves) are sold in dried form.

Market survey is an efficient means of acquiring data on local values and conservation status of indigenous species $[11,12]$. However, an understanding of the market profile, socioeconomic attributes influencing trade, species traded and impact of trade on plant pollution is critical for effective resource management [13].

The present survey aims at documenting the common medicinal plants sold in some local markets in Abeokuta, Nigeria as well as their medicinal values, local names, parts used and dosage forms.

\section{MATERIALS AND METHODS}

\section{Study area}

The study was carried out in Omida, Itoku, Adatan, Kuto and Lafenwa markets in Abeokuta, Ogun State, Nigeria. Abeokuta is located within longitude $3^{0} 21^{\prime}$ East and latitude $7^{0} 11^{\prime}$ North. The city of Abeokuta covers the geopolitical areas of Abeokuta North and Abeokuta South Local Government Areas of the State. The Yorubas are the main ethnic group in the area but the original settlers are the Egbas who founded the historic city. The dialectical groups in the area include Egbado, ljebu, Egba, Remo, Oyo (Owu), Awori, Ikale and llaje. The people are known for traditional arts, carving and sculpturing. Abeokuta is surrounded by large mass of rocks and has a population of about one million people.

\section{Survey}

This ethnomedicinal survey was conducted in October 2008 to obtain pertinent information about medicinal plants traded in the area. Ethnomedicinal data collected was based on oral interview with the aid of a structured questionnaire and only data from willing respondents were documented. It was observed that a majority of the medicinal plant traders were women. However, the responses of some the traders in the course of the interviews were unfruitful as they refused to give details of their average income, duration of trade, mode of collection and the problems encountered during sourcing of medicinal plants. They were very forthcoming in giving details of the local 
names, medicinal use, method of preparation, parts used, and dosages of the medicinal plants they sell. All the herbal medicines were prepared by either decoction or maceration.

\section{Collection and Identification of plant samples}

Plant samples were collected from five randomly selected markets in Abeokuta, Ogun State after the oral interviews with the traders. The plant samples were immediately labelled with their local names with which they were purchased and placed in clean dry baskets. They were later transferred with polythene bags to the point of identification. Most of the plant samples were identified by one of the co-authors of this work (MI). Other samples were identified using their local names and standard texts $[14,15]$.

Samples of the plants were kept in the herbarium of the Department of Plant Biology and Biotechnology, University of Benin.

\section{RESULTS}

The survey revealed a total of 60 commonly used plants distributed into 56 genera belonging to 31 families used among the people of Abeokuta in traditional healthcare. The plants are listed alphabetically in order of family names (Table 1a-e). Other information relating to their local names, medicinal uses, method of preparation, parts used and dosages were also documented.

\section{DISCUSSION}

The collection of minor forest products or non-timber forest products is the main source of the economy of tribal people [16]. The traders depend on the sale of medicinal plants for their source of income and livelihood.

Most of the plant materials in the market were in dried forms and sold singly or in combination with other plants ("concoctions") to the public. The concoctions consisted of chopped root and barks and fresh leaves and fruits and were prepared either by decoction, tincture or through maceration. Some of the medicinal plants were also in powdered form or in solutions and administered as such. Most of the remedies were prepared from a single plant source, e.g., Alstonia boonei (for treating malaria and body pain), Garcinia kola (for body pain relief), and Ficus capensis (as blood booster); a few others were in combination with other common plants such as Cassia sieberiana with Allium sativum and Eugenia aromatic(for dysentery). The concoctions were also generally provided on demand rather than prepared in advance, in order to prevent spoilage.

The major medicinal plants traded in the area were derived from trees and this accords with an earlier report about the predominance of tree species in Bachama ethnomedicine [17]. The collected medicinal plants were found to be very common among the people and were used in traditional healthcare for a variety of disease conditions such as malaria, typhoid, jaundice, hyperthermia, skin irritations, dysentery, anaemia, gonorrhea, cough, measles, hypertension and fibroid.

It was observed that the majority of medicinal plant traders were women (64.29 \%) between the age group of $40-60$ years. Young girls make up $28.5 \%$ within the age group of 17 26 years of the traders visited while $7.14 \%$ were men in the age group of $28-38$ years. The former accords with earlier reports [11] that the women constitute the major traders of medicinal plant materials.

\section{CONCLUSION}

The use of herbal medicine has always been part of human culture, including African culture. The rising demand for medicinal plants has led to increased pressure on wild plant populations and shrinking habitats. It is obvious that the bulk of the plants traded in the local markets are sourced from the wild which could result in local extinction. There 
Table 1a: Enumeration of plants commonly used by the people of Abeokuta in traditional health care (Family alphabets A - B)

\begin{tabular}{|c|c|c|c|c|c|}
\hline Family /Taxon & Voucher no. & Local name & Medicinal use & Part used & Dosage \\
\hline ALLIACEAE & & & & & \\
\hline Allium ascalonicum L. & BDHS 158 & Alubosa elewe & $\begin{array}{l}\text { Stomachic and } \\
\text { stop vomiting }\end{array}$ & $\begin{array}{l}\text { Root and } \\
\text { leaves }\end{array}$ & $\begin{array}{l}1 \text { small cup } 3 \text { times } \\
\text { daily for } 1 \text { week. }\end{array}$ \\
\hline Allium sativum $\mathrm{L}$. & BDHS 107 & Alubosa ayu & $\begin{array}{l}\text { For stroke and } \\
\text { pain in the eye }\end{array}$ & Root & $\begin{array}{l}\text { The extracted juice } \\
\text { by chewing is } \\
\text { swallowed for as } \\
\text { long as symptoms } \\
\text { persist. }\end{array}$ \\
\hline $\begin{array}{l}\text { APOCYNACEAE } \\
\text { Rauwolfia vomitoria } \\
\text { Afzel. }\end{array}$ & BDHS 149 & Asofeyeje & Anti insomnia & Root & $\begin{array}{l}1 \text { small cup once } \\
\text { daily(preferably } \\
\text { evening) for } 3 \text { days. }\end{array}$ \\
\hline $\begin{array}{l}\text { Alstonia boonei De } \\
\text { Wild. }\end{array}$ & BDHS 131 & Awun & $\begin{array}{l}\text { Anti malaria } \\
\text { and body pain } \\
\text { reliever }\end{array}$ & Bark & $\begin{array}{l}2 \text { cups } 3 \text { times daily } \\
\text { for } 2 \text { weeks. }\end{array}$ \\
\hline $\begin{array}{l}\text { ANACARDIACEAE } \\
\text { Lannea egregia Engl. } \\
\text { \& K. Krause }\end{array}$ & BDHS 128 & Eki dan & $\begin{array}{l}\text { For blood } \\
\text { supply }\end{array}$ & Bark & $\begin{array}{l}2 \text { tablespoons twice } \\
\text { daily for adults / } 2 \\
\text { teaspoons once } \\
\text { daily for children for } \\
1 \text { week. }\end{array}$ \\
\hline $\begin{array}{l}\text { Lannea welwitschii } \\
\text { (Hiern) Engl. }\end{array}$ & BDHS 142 & Orira & $\begin{array}{l}\text { Purgative and } \\
\text { anti purgative }\end{array}$ & Bark & $\begin{array}{l}1 \text { small cup once } \\
\text { daily for } 2 \text { days. }\end{array}$ \\
\hline $\begin{array}{l}\text { Anacardium } \\
\text { occidentale L. } \\
\text { ARACEAE }\end{array}$ & BDHS 115 & Kaju & $\begin{array}{l}\text { For oral } \\
\text { infection }\end{array}$ & Bark & $\begin{array}{l}1 \text { small cup once } \\
\text { daily for } 10 \text { days. }\end{array}$ \\
\hline $\begin{array}{l}\text { Anchomanes } \\
\text { difformis (Blume) } \\
\text { Engl. } \\
\text { AMARYLLIDACEAE }\end{array}$ & BDHS 122 & Langbodo & Anti hypertamia & Root & $\begin{array}{l}1 \text { small cup once } \\
\text { daily }\end{array}$ \\
\hline Curculigo pilosa Engl. & BDHS 120 & Epa kun & For gonorrhoea & Root & $\begin{array}{l}1 \text { small cup once } \\
\text { daily }\end{array}$ \\
\hline $\begin{array}{l}\text { Crinum jagus } \\
\text { (Thomson) Dandy }\end{array}$ & BDHS 114 & Isumeri & $\begin{array}{l}\text { For asthma and } \\
\text { tuberculosis }\end{array}$ & $\begin{array}{l}\text { Root and } \\
\text { leaves }\end{array}$ & $\begin{array}{l}1 \text { small cup once } \\
\text { daily (can cause } \\
\text { vomiting if taken in } \\
\text { excess). }\end{array}$ \\
\hline $\begin{array}{l}\text { ARISTOLOCHIACEA } \\
\text { E } \\
\text { Aristolochia repens } \\
\text { Mill. } \\
\text { ANNONACEAE }\end{array}$ & BDHS 160 & Ako igun & For deworming & Root & $\begin{array}{l}1 \text { teaspoon } 3 \text { times } \\
\text { daily for } 3 \text { days. }\end{array}$ \\
\hline $\begin{array}{l}\text { Uvaria afzelii Sc. } \\
\text { Elliot }\end{array}$ & BDHS 153 & Gbogbonse & For any ailment & Root & 1 cup once daily. \\
\hline $\begin{array}{l}\text { Uvaria chamae P. } \\
\text { Beauv. }\end{array}$ & BDHS 140 & Eeruju & Anti malaria & Leaves & $\begin{array}{l}1 / 2 \text { stainless steel cup } \\
\text { twice daily for } 2 \\
\text { weeks. }\end{array}$ \\
\hline $\begin{array}{l}\text { Xylopia aethiopica } \\
\text { (Dunal) A. Rich. }\end{array}$ & BDHS 125 & $\begin{array}{l}\text { Eeru awon ri n } \\
\text { ka }\end{array}$ & $\begin{array}{l}\text { For skin } \\
\text { irritation }\end{array}$ & Seed & $\begin{array}{l}1 \text { small cup once } \\
\text { daily }\end{array}$ \\
\hline $\begin{array}{l}\text { BORAGINACEAE } \\
\text { Heliotropium indicum } \\
\text { L. }\end{array}$ & BDHS 138 & Atapari-obuko & $\begin{array}{l}\text { Body pain } \\
\text { reliever }\end{array}$ & Root & $\begin{array}{l}1 \text { small cup } 3 \text { times } \\
\text { daily }\end{array}$ \\
\hline
\end{tabular}


Table 1b: Enumeration of plants commonly used by the people of Abeokuta in traditional health care (Family alphabets $\mathrm{C}-\mathrm{E}$ )

\begin{tabular}{|c|c|c|c|c|c|}
\hline Family /Taxon & Voucher no. & $\begin{array}{l}\text { Local } \\
\text { name }\end{array}$ & Medicinal use & Part used & Dosage \\
\hline $\begin{array}{l}\text { CUCURBITACEAE } \\
\text { Citrullus colocynthus } \\
\text { Schrad }\end{array}$ & BDHS 106 & Baara & For dysentery & Fruit & $\begin{array}{l}1 / 2 \text { stainless steel } \\
\text { cup twice daily for } \\
3 \text { days. }\end{array}$ \\
\hline $\begin{array}{l}\text { Adenopus breviflorus } \\
\text { Bth. }\end{array}$ & BDHS 113 & Tagiri & For measles & Fruit & $\begin{array}{l}\text { The fruits are } \\
\text { placed in the } \\
\text { patient's room for } \\
\text { as long as } \\
\text { symptoms persist. }\end{array}$ \\
\hline $\begin{array}{l}\text { CONNARACEAE } \\
\text { Agelaea obliqua (P. } \\
\text { Beauv.) Baill. }\end{array}$ & BDHS 145 & Irohin & $\begin{array}{l}\text { As an anti - } \\
\text { convulsant }\end{array}$ & Root & $\begin{array}{l}1 \text { teaspoon before } \\
\text { eating (carry the } \\
\text { victim because } \\
\text { he/she will be too } \\
\text { weak after the } \\
\text { herbal remedy is } \\
\text { administered) }\end{array}$ \\
\hline Cnestis ferruginea DC. & BDHS 123 & $\begin{array}{l}\text { Gboyin } \\
\text { gboyin }\end{array}$ & Aphrodisiac & Root & $\begin{array}{l}1 \text { small cup once } \\
\text { daily }\end{array}$ \\
\hline $\begin{array}{l}\text { COMBRETACEAE } \\
\text { Terminalia } \\
\text { avicennioides Guill. \& } \\
\text { Perr. }\end{array}$ & BDHS 144 & Idi & $\begin{array}{l}\text { For skin } \\
\text { irritation }\end{array}$ & Bark & $\begin{array}{l}\text { Drink in small } \\
\text { quantity to avoid } \\
\text { stomach }\end{array}$ \\
\hline $\begin{array}{l}\text { Terminalia superba } \\
\text { Engl. \& Diels. }\end{array}$ & BDHS 103 & Afara & $\begin{array}{l}\text { Anti malaria and } \\
\text { for measles }\end{array}$ & Bark & $\begin{array}{l}1 \text { small cup for } \\
\text { adults twice daily / } \\
2 \text { teaspoons for } \\
\text { children twice } \\
\text { daily }\end{array}$ \\
\hline $\begin{array}{l}\text { DIOSCOREACEAE } \\
\text { Dioscorea cayenensis } \\
\text { Lam. }\end{array}$ & BDHS 109 & Apepe & $\begin{array}{l}\text { For blood } \\
\text { supply }\end{array}$ & Bark & $\begin{array}{l}\text { Administer in } \\
\text { small quantity for } \\
1 \text { week. }\end{array}$ \\
\hline $\begin{array}{l}\text { EUPHORBIACEAE } \\
\text { Croton peduliflorus } \\
\text { Hutch. }\end{array}$ & BDHS 129 & Iso ariwo & For cough & Seed & $\begin{array}{l}1 \text { small cup } 3 \\
\text { times daily }\end{array}$ \\
\hline $\begin{array}{l}\text { Euphorbia lateriflora } \\
\text { Schum. \& Thonn. }\end{array}$ & BDHS 126 & Enu opiri & For typhoid & Root & $\begin{array}{l}\text { Administer in } \\
\text { small quantity for } \\
2 \text { weeks. }\end{array}$ \\
\hline $\begin{array}{l}\text { Securinega virosa } \\
\text { (Roxb. Ex Wille.) Pax. } \\
\text { et Hoffm. }\end{array}$ & BDHS 112 & Iranje & Pain reliever & Leaves & $\begin{array}{l}1 / 2 \text { stainless steel } \\
\text { cup } 3 \text { times daily }\end{array}$ \\
\hline
\end{tabular}


Table 1c: Enumeration of plants commonly used by the people of Abeokuta in traditional health care (Family alphabet F)

\begin{tabular}{|c|c|c|c|c|c|}
\hline Family /Taxon & Voucher no. & Local name & Medicinal use & Part used & Dosage \\
\hline $\begin{array}{l}\text { FABACEAE } \\
\text { Acacia ataxacantha } \\
\text { DC. }\end{array}$ & BDHS 121 & Aso bo ni & $\begin{array}{l}\text { Stomachic and } \\
\text { for dysentery }\end{array}$ & Pod and seed & $\begin{array}{l}\text { Lick soup thrice } \\
\text { daily for } 1 \text { week }\end{array}$ \\
\hline Anavlocalvx & BDHS 139 & Oko aja & Aphrodisiac & Root & $\begin{array}{l}1 \text { small cup once } \\
\text { daily for } 5 \text { days. }\end{array}$ \\
\hline $\begin{array}{l}\text { oligophyllus Bak. f. } \\
\text { Cajanus cajan (L.) } \\
\text { Millsp. }\end{array}$ & BDHS 141 & Otili & For measles & Leaves & $\begin{array}{l}1 \text { small cup } 3 \\
\text { times daily }\end{array}$ \\
\hline $\begin{array}{l}\text { Calliandra } \\
\text { haematocephala } \\
\text { Hassk. }\end{array}$ & BDHS 136 & Tude & $\begin{array}{l}\text { Stomachic and } \\
\text { for ringworm } \\
\text { treatment }\end{array}$ & Root & $\begin{array}{l}1 \text { small cup for } \\
\text { adults once daily } \\
\text { / } 1 \text { teaspoon for } \\
\text { children once } \\
\text { daily } \\
1 \text { small cup }\end{array}$ \\
\hline $\begin{array}{l}\text { Cassia sieberiana } \\
\text { DC. }\end{array}$ & BDHS 156 & Aridan tooro & For dysentery & Root & $\begin{array}{l}\text { taken only in the } \\
\text { morning for } 2 \\
\text { days. }\end{array}$ \\
\hline $\begin{array}{l}\text { Cynometra manii } \\
\text { Oliver }\end{array}$ & BDHS 134 & Eku & $\begin{array}{l}\text { To suppress } \\
\text { swelling on the } \\
\text { cheeks }\end{array}$ & Seed & $\begin{array}{l}\text { Use poultice to } \\
\text { massage the } \\
\text { cheek. }\end{array}$ \\
\hline $\begin{array}{l}\text { Daniella oliveri } \\
\text { (Rolfe.) Hutch. \& }\end{array}$ & BDHS 132 & lya & For rashes & Bark & $\begin{array}{l}1 \text { teaspoon for } \\
\text { babies }\end{array}$ \\
\hline Dalz & BDHS 159 & Ogbogbo & For dysentery & Bark & No dosage \\
\hline $\begin{array}{l}\text { Detarium } \\
\text { microcarpum }\end{array}$ & BDHS 137 & Dasa & d sight & & \\
\hline Dioclea secandens & מנד & Dasd & $\begin{array}{l}\text { and to stop } \\
\text { excessive } \\
\text { menstrual flow } \\
\text { (Anti } \\
\text { menorrhagia) }\end{array}$ & Leaves & No dosage \\
\hline $\begin{array}{l}\text { Erythrophleum } \\
\text { suaveolens (Guill. } \\
\text { \&Perr.) Brenan }\end{array}$ & BDHS 101 & Obo & $\begin{array}{l}\text { For skin } \\
\text { irritation }\end{array}$ & Bark & $\begin{array}{l}\text { Concoction is } \\
\text { used to bath } \\
\text { twice daly. }\end{array}$ \\
\hline $\begin{array}{l}\text { Indigofera pilosa } \\
\text { Poir. }\end{array}$ & BDHS 157 & Ka se & Anti malaria & Bark & $\begin{array}{l}1 \text { small cup twice } \\
\text { daily }\end{array}$ \\
\hline $\begin{array}{l}\text { Lonchocarpus } \\
\text { cyanescens } \\
\text { (Schum. \& Thonn.) }\end{array}$ & BDHS 152 & Elu & For rashes & Root & $\begin{array}{l}1 \text { small cup once } \\
\text { daily for adults } \\
\text { and bath once } \\
\text { daily for children }\end{array}$ \\
\hline $\begin{array}{l}\text { Tetrapleura } \\
\text { tetraptera (Schum } \\
\text { \& Thonn.)Taub }\end{array}$ & BDHS 124 & Aridan & Anti convulsant & Fruit & $\begin{array}{l}2 \text { small cups for } \\
\text { adults / } 2 \\
\text { teaspoons for } \\
\text { children twice } \\
\text { daily }\end{array}$ \\
\hline
\end{tabular}


Iduet al

Table 1d: Enumeration of plants commonly used by the people of Abeokuta in traditional health care (Family alphabets $\mathrm{G}$ - O)

\begin{tabular}{|c|c|c|c|c|c|}
\hline Family /Taxon & Voucher no. & Local name & $\begin{array}{l}\text { Medicinal } \\
\text { use }\end{array}$ & Part used & Dosage \\
\hline $\begin{array}{l}\text { GRAMINEAE } \\
\text { Bambusa vulgaris L. } \\
\text { GUTTIFERAE }\end{array}$ & BDHS 111 & Oparun & For measles & Leaves & $\begin{array}{l}1 / 2 \text { stainless cup } 3 \\
\text { times daily }\end{array}$ \\
\hline Garcinia kola Heckel. & BDHS 116 & Orogbo & $\begin{array}{l}\text { Body pain } \\
\text { reliever }\end{array}$ & Bark & $\begin{array}{l}1 \text { small cup once } \\
\text { daily (for adults } \\
\text { only) }\end{array}$ \\
\hline $\begin{array}{l}\text { LYTHRACEAE } \\
\text { Lawsonia inermis L. }\end{array}$ & BDHS 118 & Laali & For typhoid & Leaves & $\begin{array}{l}1 / 2 \text { stainless steel } \\
\text { cup } 3 \text { times daily }\end{array}$ \\
\hline $\begin{array}{l}\text { MORACEAE } \\
\text { Bosqueia angolensis } \\
\text { Ficalho. }\end{array}$ & BDHS 135 & Saworo & $\begin{array}{l}\text { For blood } \\
\text { supply }\end{array}$ & Bark & No dosage \\
\hline $\begin{array}{l}\text { Treculia africana } \\
\text { Decne. } \\
\text { Moraceae }\end{array}$ & BDHS 117 & Afon & $\begin{array}{l}\text { For skin } \\
\text { irritation }\end{array}$ & Root & $\begin{array}{l}1 \text { teaspoon for } \\
\text { children } 3 \text { times } \\
\text { daily }\end{array}$ \\
\hline Ficus capensis Thunb. & BDHS146 & Opoto & $\begin{array}{l}\text { For blood } \\
\text { supply }\end{array}$ & Leaves & $\begin{array}{l}1 / 2 \text { stainless steel } \\
\text { cup } 3 \text { times daily } \\
\text { for adults and } 1 \\
\text { teaspoon } 3 \text { times } \\
\text { daily for children }\end{array}$ \\
\hline $\begin{array}{l}\text { MELIACEAE } \\
\text { Khaya ivorensis A. } \\
\text { Chev } \\
\text { MENISPERMACEAE }\end{array}$ & BDHS 104 & Oganwo & $\begin{array}{l}\text { For blood } \\
\text { supply }\end{array}$ & Bark & $\begin{array}{l}1 \text { small cup once } \\
\text { daily }\end{array}$ \\
\hline $\begin{array}{l}\text { Sphenocentrum } \\
\text { jollyanum Pierre }\end{array}$ & BDHS 150 & Akerejukpon & $\begin{array}{l}\text { Anti malaria } \\
\text { and typhoid }\end{array}$ & Root & $\begin{array}{l}1 \text { stainless steel } \\
\text { cup twice daily } \\
\text { for } 2 \text { weeks. }\end{array}$ \\
\hline $\begin{array}{l}\text { MYRTACEAE } \\
\text { Syzygium guineense } \\
\text { (Wild.) DC. }\end{array}$ & BDHS 133 & Ori & $\begin{array}{l}\text { Purgative } \\
\text { and anti } \\
\text { purgative }\end{array}$ & Bark & $\begin{array}{l}\text { I small cup once } \\
\text { for adults / } 2 \\
\text { teaspoons once } \\
\text { daily for children }\end{array}$ \\
\hline $\begin{array}{l}\text { OCHNACEAE } \\
\text { Lophira alata Banks ex } \\
\text { F. Gaertn.f. } \\
\text { OLEACEAE }\end{array}$ & BDHS 148 & Ponhan & For typhoid & Bark & $\begin{array}{l}1 \text { small cup } 3 \\
\text { times daily }\end{array}$ \\
\hline $\begin{array}{l}\text { Schrebera arborea } \\
\text { A.Chev. }\end{array}$ & BDHS 127 & Opele & $\begin{array}{l}\text { For skin tear } \\
\text { on a child's } \\
\text { head }\end{array}$ & Seed & $\begin{array}{l}\text { Place } 9 \text { seeds } \\
\text { on male and } 7 \text { on } \\
\text { female child (as } \\
\text { seed closes the } \\
\text { child's head } \\
\text { heals) }\end{array}$ \\
\hline
\end{tabular}


Iduet al

Table 1e: Enumeration of plants commonly used by the people of Abeokuta in Traditional health care (Family alphabets $\mathrm{P}$ - Z)

\begin{tabular}{|c|c|c|c|c|c|}
\hline Family /Taxon & Voucher no. & Local name & $\begin{array}{l}\text { Medicinal } \\
\text { use }\end{array}$ & Part used & Dosage \\
\hline $\begin{array}{l}\text { PAPAVERACEAE } \\
\text { Argemone mexicana } \\
\text { L. } \\
\text { PHYLLANTHACEAE }\end{array}$ & BDHS 143 & $\begin{array}{l}\text { Ma fo wo } \\
\text { kan omo mi }\end{array}$ & For measles & Leave & $\begin{array}{l}\text { To be used in } \\
\text { small quantity }\end{array}$ \\
\hline $\begin{array}{l}\text { Bridelia atroviridis } \\
\text { Mull. Arg. }\end{array}$ & BDHS 102 & Arasa & For eczema & Leaves & $\begin{array}{l}\text { Rub on } \\
\text { affected part } \\
\text { for as long as } \\
\text { conditions } \\
\text { remains }\end{array}$ \\
\hline $\begin{array}{l}\text { POACEAE } \\
\text { Cymbopogon citratus } \\
\text { (DC.) Stapf. }\end{array}$ & BDHS 147 & Ewe tea & Anti malaria & Leaves & $\begin{array}{l}2 \text { small cups } 3 \\
\text { times daily for } \\
2 \text { weeks. }\end{array}$ \\
\hline $\begin{array}{l}\text { PERIPLOCACEAE } \\
\text { Mondia whitei } \\
\text { (Hook.f.) Skeels }\end{array}$ & BDHS 105 & Isirigun & $\begin{array}{l}\text { For } \\
\text { deworming }\end{array}$ & Root & $\begin{array}{l}1 \text { teaspoon } 3 \\
\text { times daily (for } \\
\text { children) }\end{array}$ \\
\hline $\begin{array}{l}\text { RUBIACEAE } \\
\text { Nauclea latifolia Sm. }\end{array}$ & BDHS 154 & Egbesi & $\begin{array}{l}\text { For jaundice } \\
\text { (yellow fever) }\end{array}$ & Root & $\begin{array}{l}1 \text { small cup } \\
\text { once daily }\end{array}$ \\
\hline $\begin{array}{l}\text { STERCULIACEAE } \\
\text { Theobroma cacao L. }\end{array}$ & BDHS 130 & Koko & $\begin{array}{l}\text { For blood } \\
\text { supply }\end{array}$ & Bark & $\begin{array}{l}1 \text { small cup } 3 \\
\text { times daily }\end{array}$ \\
\hline $\begin{array}{l}\text { Nesogordonia } \\
\text { papaverifera (A. } \\
\text { Chev.) R. Capuron }\end{array}$ & BDHS 108 & Oro & For fibroid & Bark & $\begin{array}{l}1 \text { small cup } \\
\text { once daily }\end{array}$ \\
\hline $\begin{array}{l}\text { Cola gigantea } A . \\
\text { Chev. }\end{array}$ & BDHS 151 & Oporoporo & $\begin{array}{l}\text { For blood } \\
\text { supply }\end{array}$ & Leaves & $\begin{array}{l}1 \text { small cup } 3 \\
\text { times daily for } \\
1 \text { week. }\end{array}$ \\
\hline $\begin{array}{l}\text { SAPINADIACEAE } \\
\text { Lecaniodiscus } \\
\text { cupanioides Planch. } \\
\text { ex Benth. }\end{array}$ & BDHS 161 & Aka & $\begin{array}{l}\text { To heal } \\
\text { fractures and } \\
\text { wounds of the } \\
\text { leg }\end{array}$ & Root & $\begin{array}{l}\text { Rub on } \\
\text { affected parts. }\end{array}$ \\
\hline $\begin{array}{l}\text { ZINGIBERACEAE } \\
\text { Aframomum } \\
\text { melegueta K. Schum. }\end{array}$ & BDHS 119 & Ata ire & For measles & Leaves & $\begin{array}{l}1 \text { small cup } 3 \\
\text { times daily for } \\
2 \text { weeks. }\end{array}$ \\
\hline $\begin{array}{l}\text { Zingiber officinale } \\
\text { Rosc. }\end{array}$ & BDHS 110 & Atalekopa & For typhoid & Root & $\begin{array}{l}\text { Eat hot once } \\
\text { daily. }\end{array}$ \\
\hline
\end{tabular}


is, therefore, the need to encourage domestication and cultivation of medicinal plants as well as put in place conservation measures to ensure sustainable source of plant materials.

\section{REFERENCES}

1. Fabeku, PO. Traditional Medicine: the art, ways and practice. In: Odugbemi, T, editor, Outlines and Pictures of Medicinal Plants from Nigeria. University of Lagos Press; 2006. p.13-24.

2. Neumann RR, Hirsch E. Commercialization of NonTimber Forest Products: Review and Analysis for Research, Indonesia: CIFOR; 2000. 176p

3. Yesilada E. Past and future contributions of traditional medicine in the healthcare system of the Middle East. J of Ethnopharmacol, 2005; 100: 135-137.

4. Saed $M$, Arshad $M$, Ahmed $E$, Ishaque $M$. Ethnophytotherapies for the treatment of various diseases by the local people of selected areas of N.W.F.P. Parkistan J Biol Scien 2004; 7:1104-1108.

5. Sheldon JW, Berlick. MJ, Laird SA. Medicinal plants: can utilization and Conservation Coexist? Advances in Economic Botany, 1997; 12:1-104

6. Alves RRN, Rosa IL. Why study the use of animal products in traditional medicines? J Ethnobiol Ethnomed 2005; 1: 1-5.

7. Omwuliri FC, Wonang DL. Studies on the combined antibacterial action of ginger (Zingiber officinale L.) and garlic (Allium sativum L.) on some bacteria. Nig J Botany 2005, 18: 224228
8. Hanazki N, Tamishoro JY, Leitao- Filho H, Gegossi A. Diversity of Plant Uses in Caicaras Communities from the atlantic forest coast, Brazil. Biodiversity and Conservation, 2000; 9: 597-615

9. Pei SJ. Ethnobotanical approaches of Traditional medicine studies: Some experiences from Asia. Pharmaceutical Biol 2001; 39: 74-79.

10. W.H.O. World Health Organisation guidelines on good agricultural and collection practices (GACP) for medicinal plants 2003. 80p

11. Idu M, Osawaru M, Orhue ES. Medicinal plants in some local markets in Benin City, Nigeria. Ethnobotany 2005; 17: 118-122

12. Martin G. Ethnobotany: London: Chapman and Hall. 1995. 296p

13. Botha J, Witkowski ETF, Shackleton CM. Market Profiles and Trade in medicinal plants in the lowveld, South Africa. Environ Conserva 2004; 31: 38-46.

14. Odugbemi $T$, Akinsulire O. Medicinal plants by species names In: Odugbemi, $T$, editor: Outlines and pictures of Medicinal Plants from Nigeria. University of Lagos Press; 2006. p.73161

15. Akobundu IO, Agyakwa CW. A Handbook of West African Weeds Ibadan: International Institute of Tropical Agriculture; $1998.564 p$

16. Kumar A, Jain SK. Plant products in some tribal markets of central India. Economic Botany, 2002; 56: 242-245.

17. Idu $M$, Gill LS, Omonhinmin CA, Ejale $A$. Ethnomedicinal uses of trees among Bachama tribe of Adamawa state, Nigeria. Indian J. Trad. Knowledge. 2006; 5: 273-278. 